\title{
FENOMENOLOGÍA TRASCENDENTAL Y SCIENTIA TRASCENDENS: DOS VERSIONES DE LA INTENCIONALIDAD
}

\author{
Transcendental Phenomenlogy and Scientia Trascendens: Two Conceptions of the Intentionality
}

Fenomenologia Transcendental e Scientia Trascendens: Duas Versões da Intencionalidade

Julio CéSAR Vargas Bejarano

\begin{abstract}
Resumen: Este trabajo confronta dos versiones de la intencionalidad, de Duns Escoto y de Edmund Husserl. Esta confrontación permite establecer en qué medida la filosofía de Duns Escoto, la scientia trascendens, enriquece la concepción fenomenológica de la intencionalidad, y permite determinar si la filosofía de Escoto contiene una 'fenomenología de la verdad'. En el desarrollo del trabajo se muestra que, a pesar de algunas convergencias importantes con respecto a la intencionalidad, Escoto y Husserl tienen dos concepciones muy distintas del fenómeno o de la presencia; para el primero el objeto intencional se ofrece mediante las especies inteligibles, para el segundo a través de la fenomenalización. La investigación muestra que el mayor punto de divergencia está en la concepción del ser: para Escoto ser y verdad van de la mano, con independencia de los actos intencionales. Para Husserl, el sentido del ser surge de la actividad intencional de la conciencia, trascendental, determinada por los horizontes de orden temporal, mundano, intersubjetivo. Finalmente, Escoto plantea preguntas de orden metafísico, que llevan a reflexionar sobre los límites de esta disciplina. Palabras clave: Intencionalidad, acto intencional, conciencia, objeto inteligible, representación
\end{abstract}

\begin{abstract}
This essay compares intentionality in Duns Scotus and Edmund Husserl. Such comparison allows to consider up to which point Scotus's philosophy, the scientia transcendens, enriches the phenomenological concept of intentionality and allows to determine whether such philosophy contains a "phenomenology of truth". It is shown that, despite some important convergencies respect of intentionality, Scotus and Husserl have two very different conceptions of the phaenomenon or the presence; for the first one, the intentional object is offered through the intelligible species, whereas for the second this is done through phaenomenization. This research shows that the main point of divergence is the conception of being: for Scotus, being and truth go together, independantly from intentional acts. For Husserl, the meaning of being arises from the intentional activity of the transcendental conscience, determined by intersubjective, worldly, temporal horizons. Finally, Scotus states questions of metaphysical character, which lead to think about the limits of this discipline.
\end{abstract}

Keywords: Intentionality, intentional act, conscience, inteligible object, representation.

Resumen: Este trabalho confronta duas versões de intencionalidade, de Duns Escoto e de Edmund Husserl. Esta confrontação permite estabelecer em que medida a filosofía de Duns Escoto, a scientia trascendens, enriquece a concepção fenomenológica de intencionalidade, e permite determinar se a filosofía de Escoto contém uma "fenomenología da verdade”. No desenvolvimento do trabalho mostra-se que, apesar de algumas convergências importantes em relação à intencionalidade, Escoto e Husserl têm duas concepções muito diferentes do fenômeno ou da presença; para o primeiro, o objeto intencional é oferecido através de espécies inteligíveis; para o segundo, através da fenomenalização. Pesquisas mostram que o maior ponto de divergência está na concepção do ser: para Escoto, o ser e a verdade andam de mãos dadas, independentemente de atos intencionais. Para Husserl, a sensação de ser surge da atividade intencional da consciência, transcendental, determinada por horizontes de ordem temporal, mundana e intersubjetiva. Finalmente, Escoto coloca questões de ordem metafísica, que levam a refletir sobre os limites dessa disciplina.

Palavras-Chave: Intencionalidade, Ato Intencional, Consciência, Objeto Inteligível, Representación

“... se debe tomar conciencia de que la psicología escolástica mantiene una orientación a los principales problemas objetivo-noemáticos, precisamente, por no estar enfocada al psiquismo real, fluyente-dinámico, situación que favorece ampliamente el dirigir la atención a los fenómenos de la intencionalidad. Para la decisiva comprensión de este carácter fundamental de la psicología escolástica, asumo como especialmente apremiante la elaboración filosófica, más exactamente, fenomenológica, de los textos místicos, teológico-morales y estéticos de la escolástica medieval."(Heidegger, 1978, 205)
En este trabajo me propongo confrontar las versiones escotista y husserliana de la intencionalidad. La confrontación se justifica, en primer lugar, porque se trata de concepciones filosóficas que marcaron la renovación de la ontología y de la metafísica. En el primer caso, Escoto construye una nueva ontología como scientia trascendentes; en el segundo caso, la fenomenología de Husserl ofrece una compleja teoría de la intencionalidad que va desde una eidética, hasta un análisis detallado de la estructura y de la génesis de la correlación intencional. En segundo lugar, la confrontación permite apreciar dos énfasis en relación con el problema de la aprehen- 
sión intencional: de una parte, la filosofía escotista acentúa la acción del entendimiento, que accede al objeto inteligido (esse inteligibile) y con ello advierte que el sentido último de la realidad natural proviene de la dimensión sobrenatural, divina; de otra parte, la fenomenología de Husserl pone de relieve el vínculo indisociable entre sensibilidad y entendimiento, a la vez que encuentra en la experiencia vivida, en el mundo de la vida, la fuente del sentido de ser. El contraste entre estos diversos enfoques teóricos de la intencionalidad lleva a plantear la pregunta sobre la vigencia de la filosofía escotista.

La primera parte de este trabajo ofrece los rasgos centrales de la teoría escotista de la intencionalidad; la segunda parte coteja las versiones escotista y husserliana de la intencionalidad. La tercera parte reflexiona sobre la contribución de la scientia trascendens a la fenomenología trascendental.

\section{La doctrina escotista de la intencionalidad}

En conexión con los problemas ontológicos, de teoría del conocimiento y metafísicos que dejó planteados Aristóteles, la filosofía medieval se ocupa a fondo del tema de la 'intencionalidad', desde diversas perspectivas que corresponden a la teoría del conocimiento, la lógica, la metafísica, la filosofía del lenguaje y la ética. Duns Escoto ofrece una versión novedosa de la intencionalidad, cuyo influjo no se limita a la escuela escotista, sino se extiende a pensadores como Suárez y Wolff, Pierce, e implícitamente - a partir de la doctrina de la "distinción formal" - a la modernidad. ${ }^{1}$

La intencionalidad es un atributo esencial de la vida espiritual que designa la compleja relación entre el espíritu y los objetos, sean éstos reales, representados, o producto de la imaginación o fantasía; designa el modo como el espíritu aprehende el ser de los objetos reales o representados. El problema central de la intencionalidad consiste en determinar cómo puede el espíritu acceder al objeto de conocimiento, si lo hace directamente, o si lo hace mediante la intervención de una representación mental o psíquica. El punto de referencia tradicional para dar cuenta de la intencionalidad es la posición de Brentano, quien en su obra Psicología desde un punto de vista empírico afirma que el objeto intencional es in-existente en el espíritu, de manera que no es posible mostrar cómo se efectúa la referencia al objeto real, pues el conocimiento sólo puede dar cuenta de su respectivo objeto en tanto representación mental. ${ }^{2}$ No obstan-

\footnotetext{
1 Este trabajo es resultado de la investigación titulada "Univocidad e intencionalidad en el pensamiento de Duns Escoto”, 2016, adscrita a la Vicerrectoría de Investigaciones de la Universidad del Valle. La doctrina de la "distinción formal" constituye el núcleo de la ontológica escotista de la univocidad. De Muralt (2008) ha mostrado de qué manera la ontología escotista sienta los supuestos teóricos, que sirven de base a pensadores como Descartes, Spinoza, Leibniz, entre otros. Para una lectura de los vínculos entre Duns Escoto, Kant y Pierce, véase Honnefelder 1990, Boulnois, 1999.

2 En 1874, Brentano describe la intencionalidad en los siguiente términos: "Todo fenómeno mental se caracteriza por lo que los Escolásticos de la Edad Media han llamado la inexistencia intencional (o mental) de un objeto, y lo que podríamos llamar, aunque no totalmente sin ambigüedad, referencia a un contenido, dirección hacia un objeto (el cual no debe ser entendido aquí como significando una cosa), $\mathrm{u}$ objetividad inmanente. Todo fenómeno mental incluye algo
}

te, es de tener presente que si bien la tesis de que el objeto intencional in-existe en la mente, al modo de una representación, tiene una raíz escolástica, en este período de pensamiento hay una pluralidad de concepciones sobre la intencionalidad que rechazan esta mediación mental, entre ellas cabe mencionar la posición de Pedro de Juan Olivi y de Guillermo de Ockham. Y, si bien la representación es central en la teoría escotista de la intencionalidad, con todo, difiere en varios aspectos con respecto a la versión de la in-existencia mental del objeto. Para dar cuenta de la doctrina de la intencionalidad de Duns Escoto, debemos determinar, en primer lugar, cómo interpreta la referencia intencional a la realidad, de qué manera se puede garantizar la relación esencial y no meramente accidental entre el entendimiento y los objetos del mundo natural, "extra-mental".

En sus escritos juveniles, especialmente en sus Comentarios a la Isagogé, a las Categorías y al De Interpretatione de Aristóteles, Escoto construye los cimientos para su teoría de la intencionalidad, que desarrolla especialmente en la Ordinatio I, dist. 3, pars 1, en los Comentarios a la Metafísica, y en algunos pasaje de las Reportata Parisense. En sus escritos sobre lógica, Escoto se enfrenta al problema de cómo elevar esta disciplina a ciencia. Siguiendo las indicaciones de Aristóteles sobre la naturaleza de la ciencia, determina el objeto (subiectum) de la lógica. Para ello, asume como punto de referencia la distinción aviceniana entre intenciones primeras e intenciones segundas (Salinas 2016). Es de advertir que en este primer período, Escoto restringe la doctrina de la univocidad al campo del pensamiento, de la lógica, sin ampliarla a la pregunta por el ser de la realidad, por la metafísica (Salinas 2017). Tarea que lleva a cabo en su pensamiento de madurez, en donde reelabora su concepción de la intencionalidad, la cual en sus primeras investigaciones sobre lógica estaba concentrada en el tema de las intenciones segundas (Pini 2002; 2008).

Si bien Aristóteles sienta las bases de la intencionalidad (Aristóteles, 1994, V, 1021a31-1021b4; VI, 1027b 25-27), el desarrollo temático de la intentio se inicia en el pensamiento árabe. El término intentio es la traducción latina de las palabras $m a^{\prime} q u l^{\prime}$ y ' $m a^{\prime} n a^{\prime}$, que Al-Farabi utilizó para traducir el término griego 'noema', contenido conceptual del pensamiento, resultado de la acción del intelecto, referidos por Aristóteles en su obra De Interpretatione (Aristóteles, 1999, 16ª 3-18) ${ }^{3}$ Para dar cuenta de la relación con el objeto, en cuanto tal, Avicena establece la diferencia entre intenciones primeras y segundas, la cual corresponde a dos órdenes del ser: de una parte la realidad percibida, natural; de

como objeto dentro de sí mismo, aunque no todos ellos lo hacen del mismo modo. En la representación algo es representado; en el juicio algo es afirmado o negado; en el amor, amado; en el odio, odiado; en el deseo, deseado, etc." (Brentano, 1924, p. 124-125). Sin embargo, se debe tener presente que, en 1991, en el segundo volumen de su "Psicología", Brentano. reformula esta concepción, acepta las críticas de Husserl, de manera que la intencionalidad deja de ser interpretada como una mediación entre el sujeto y la realidad externa.

3 Al-Farabi, en su "Comentario" a dicha obra, sostiene que dicho contenido de pensamiento varía de acuerdo con el objeto hacia donde se dirija la atención: o bien hacia la realidad objetiva, externa, o bien hacia las propia alma y su lenguaje. En el primer caso, los objetos son externos al acto; en el segundo, mediante un acto reflexivo, el alma dirige la atención al modo como realiza los actos. Cfr. Knudsen, 1982 , p. 479 ss. 
otra parte, el campo de las idealidades lógicas, resultado de la actividad del intelecto. Mediante las primeras se efectúa el conocimiento directo de las cosas (tales como 'piedra', 'árbol') expresables mediante conceptos. El término tasawwur designa este primer nivel de las intenciones, y se tradujo al latín como imaginatio, (conception). Las intenciones segundas corresponden al conocimiento lógico (credulitas, tasdiq, assent) que se realiza mediante proposiciones, resultado de actos reflexivos: logica rationalis. En ellas, el intelecto opera sobre las intenciones primeras y mediante inferencias y deducciones accede a lo desconocido a partir de lo conocido. Las intenciones segundas conforman el campo de la lógica, las operaciones de la razón, distinto de ciencias empíricas como la psicología y las ciencias del lenguaje. En palabras de Avicena: "En efecto, el objeto de la lógica, como sabes, son las intenciones inteligidas en segundo lugar, ellas se aplican a las intenciones inteligidas en primer lugar, en tanto que por ellas se llega de lo conocido a lo desconocido" (Avicena, 1977, 2. Traducción de H. Salinas).

Esta diferencia resulta decisiva para deslindar el campo de la lógica, conceptual y universal, conformado por intenciones segundas, del campo de ciencias empíricas, de la psicología y de las ciencias del lenguaje. Por ello, la noética medievalista, de Tomás de Aquino y de Escoto, no cae ni en un sermocinalismo, según el cual el objeto de la lógica es el discurso, la palabra hablada, la gramática, ni en un psicologismo, tal y como se entiende en el siglo XIX. Aquí encontramos un aspecto en común entre Escoto y Husserl, pues ambos delimitan el campo de la lógica con respecto a las ciencias empíricas e intentan constituir la lógica como ciencia, ciencia común a las demás ciencias, cuya base está en la evidencia. Para alcanzar este propósito, el tema de la intencionalidad resulta decisivo, pues permite determinar cómo el espíritu aprehende objetos reales, existentes, y los diferencia de otros tipos de objeto, recuerdos, fantasías, entre otros.

Para dar cuenta de la intencionalidad, en el pensamiento de Escoto, introducimos su concepción del 'objeto' intencional y establecemos sus vínculos con la ontología. El doctor sutil distingue dos tipos de conocimiento: a) el conocimiento intuitivo, o del objeto absoluto, tal y como se ofrece aquí y ahora y b) el conocimiento abstractivo, cuyo objeto no es el ente en cuanto existente, sino en cuanto intencional. Aquí nos ocupamos de este último, pues es el más representativo para la concepción ontológica de Escoto, el conocimiento intuitivo no sería propio del ser humano en su estado actual; el relevante es el abstractivo, base del conocimiento científico.

Sobre la base de que la percepción no puede acceder a la esencia del objeto singular, sino que ella ofrece un conocimiento limitado de lo existente, el doctor sutil renueva la concepción del objeto del conocimiento. Siguiendo a Aristóteles, afirma que la ciencia no se ocupa de singulares, sino que despliega su saber en el orden teórico, inteligible, de manera que la percepción, que informa sobre la existencia actual del objeto, no añade nada al concepto de un ente determinado. El entendimiento humano tan sólo puede dar cuenta de las realidades empíricas de un modo indirecto, a partir del ser inteligible, de la quididad. De manera que una vez accede al contenido esencial del objeto, éste subsiste en el alma, con total independencia de la realidad existente.

El objeto intencional pertenece al orden objetivo, cuenta con propiedades formales que cualquier tipo de objeto comparte, objeto inteligido, correlato del pensamiento. El objeto inteligido tiene un modo de ser peculiar, es concebido 'en tanto que', 'en un cierto aspecto' (esse secundum quid). Para referirse a él, Escoto utiliza la expresión "disminuido", lo cual no quiere decir que el objeto quede reducido, ni que pierda tamaño o calidad, sino que se aprehende tal como es, esencialmente. Esta aprehensión simple, conceptual, equivale a la "primera intención", representación, semejante y verdadera (vera similitudo obiecti), del objeto existente, absoluto (esse simpliciter. Scotus, 1960, p. 377) ${ }^{4}$. El pensamiento tiende hacia su objeto, lo aprehende. Y el objeto inteligido cuenta con un modo de ser propio, que antecede al pensamiento finito. En palabras de Escoto:

La potencia cognitiva no sólo debe recibir las especies de su objeto, sino también debe tender mediante su acto hacia el objeto. Y esto segundo es más esencial para la potencia, pues el primero se requiere por cuenta de la imperfección de la potencia. Y el objeto es más primordialmente un objeto porque la (potencia) misma tiende hacia él, que por porque en él se impriman las especies (Scotus, 1997, $I V, 290) .^{5}$

Aquí reside un aspecto central de la intencionalidad escotista, según la cual la intencionalidad no se limita a la dimensión pasiva, sino que se caracteriza especialmente por su carácter activo, por la tendencia del entendimiento hacia el objeto. Más aún, esta tendencia no sólo involucra al entendimiento, sino que está presente en toda la actividad anímica, desde la sensibilidad hasta la volición. Esta tesis la retoma Escoto de Odón Rigaud (1210-1275) y Pedro de Juan Olivi (1247-1296).

4 En relación a la similitud del objeto intencional con la cosa extramental, existente, cabe señalar que en sus escritos de lógica, Escoto desarrolla una concepción de la intencionalidad según la cual el entendimiento - valiéndose de las categorías - tan sólo accede al ser de las cosas, a la sustancia, de un modo accidental. De manera que la lógica alcanza el estatuto de ciencia, gracias a la univocidad del pensamiento categorial, garante de la univocidad a nivel de la predicación. En el orden lógico, el objeto intencional tiene un carácter categorial o conceptual. En este primer período, el doctor sutil no entiende aún la filosofía como ontología, scientia trascendens, el pensamiento no alcanza a aprehender, unívocamente, el orden real, sustancial (Salinas 2017).

5 "Nam potentia cognitiva non tantum habet recipere speciem obiecti, sed etiam tendere per actum suum in obiectum. Et istud secundum est essentialius potentiae, quia primum requiritur propter imperfectionem potentiae. Et obiectum principalius est obiectum quia in ipsum tendit potentia, quam quia imprimit speciem."

6 En oposición al carácter meramente potencial de la intentio, que la limita a la voluntad o a su objeto volitivo (Pedro Lombardo), Rigaud sostiene que la intentio es el acto mediante el cual el alma dirige su atención y acción al objeto, la intentio es el rasgo característico del alma que participa en todas sus facultades, desde la afectividad, pasando por el intelecto, hasta la volición. La novedad de Rigaud consiste en afirmar que la apropiación del objeto intencional depende menos de condiciones externas, por ejemplo del medio, que de la acción o tendencia anímica. En este sentido, Olivi desarrolla las tesis de Rigaud, y deniega el carácter de representación del objeto intencional. El acceso al objeto está determinado primordialmente por la actividad intencional, sin que esto signifique que la imagen mental equivalga a la especie inteligible, 
Para Escoto el conocimiento es relativo a la estructura óntica de los objetos. En el caso de la percepción, los objetos cuentan con un componente material y con una dimensión esencial. En correspondencia con esto, el conocimiento surge de la conjunción entre dos causas parciales, independientes y convergentes, una subordinante y otra subordinada, que conforman un orden esencial: entendimiento y objeto. De una parte, la sensibilidad ofrece la imagen, representación sensible o fantasma; de otra parte, el entendimiento abstrae a partir de la imagen, da un salto de lo particular a lo universal, accede a las especies inteligibles. En ellas el objeto sensible aparece transpuesto en un nuevo orden intencional, de carácter universal y esto mediante la especie intelectiva. No obstante, trasponer no es constituir o construir, sino representar, pero no como intención vacía, sino en el sentido de mostrar o presentar. Que el entendimiento accede a la especie intelectiva, quiere decir que representa el objeto desde un punto de vista abstracto, desprovisto de condiciones empíricas, por ejemplo, el caballo representado en general, como un individuo, o parte de un universal, esto es, desprovisto de relación con el paisaje en donde se encuentra, y de cualidades propias como el peso, la calidad del pelaje, entre otras.

Escoto acoge la distinción aristotélica entre entendimiento agente, cuya función es pasar del orden sensible (de la imagen) al inteligible, y el entendimiento pasivo, que recibe, conserva y actualiza mediante el recuerdo las especies intelectivas. El conocimiento es resultado del concurso de dos causas independientes: una primordial, el entendimiento y la otra subordinada, el objeto.

Las especies inteligibles afectan el entendimiento pasivo, informan la imagen, pero en este nivel no contamos aún con el objeto inteligido como tal, para ello hace falta la referencia intencional. Las especies inteligibles contribuyen a la comprensión del objeto, permiten representarlo en el orden lógico, como universal, perteneciente a tal género, con tales o cuales atributos; sin embargo, no son ni la representación psíquica, subjetiva, ni el objeto como tal, en sí, sino un "mecanismo intelectual", en donde se hace presente el objeto inteligido (ratio formalis intellectualis). En las especies inteligibles se hace presente el objeto, pero aún no tiene lugar el acto de la intelección.

“Esto [la presencia del 'objeto cognoscitivo'] no requiere la presencia real del 'objeto en sí, sino requiere algo en donde reluce el objeto. Por eso, afirmo que la 'presencia de lo real' del objeto es causa de la especie real y en ella está presente el objeto (...) la especie es de tal naturaleza que en ella está presente el 'objeto cognoscible', pero no de una manera efectiva o real, sino bajo el modo de ser reluciente." (Scotus, 1954, p. 366-67). ${ }^{7}$

En suma, el conocimiento cuenta con tres niveles:

o que sea base para ésta. Martínez Ruiz (2014) ofrece una exposición detallada de esta temática.

7 "et illa non requirit praesentiam realem 'obiecti in se', sed requirit aliquid in quo relucet obiectum. Dico igitur quod 'praesentia realis' obiecti est causa realis speciei, et in illa est obiectum praesens (...) species enim est talis naturae quod in ea est praesens 'obiectum cognoscibile' non effective vel realiter, sed per modum relucentis.". Véase también Perler 2001, p. 208ss. a) el objeto singular, material, punto de partida del conocimiento, que no conforma aún el objeto primordial del conocimiento

b) Las especies inteligibles o mecanismo lógico en donde se hace presente el objeto y

c) el objeto primario, conocido, el objeto inteligible, cuya existencia es 'disminuida'. (res sub ratione cognoscibilis seu repraesentati). (Scotus, 1954, p. 232, 158; Perler, 2001, p. 209).

Según lo dicho, daría la impresión de que hay una identidad entre el objeto inteligible y la 'esencia' (esse essentiae), realidad neutra, preexistente, pensada por Dios, que luego puede actualizar, (esse existentiae). Esta tesis la defendió Enrique de Gante, apoyado en Avicena. Sin embargo, Escoto afirma que en Dios los objetos no prexisten al modo de esencias, y muchos menos éstas condicionan la voluntad divina. El conocimiento tiene un carácter intencional que no consiste en acceder a las esencias, sino en acceder al objeto como correlato necesario del pensamiento, objeto inteligido (esse inteligibile).

Por tanto, digo que una cosa no tiene en la eternidad un verdadero ser de esencia o de existencia, sino que ella funda la relación ideal según un ser disminuido (deminutum) que ella posee desde la eternidad (que el verdadero ser es distinto del ser esencial y del ser de la existencia, lo expresa el libro VI de la Metafísica): si se supone que yo fuera y que conociera una rosa por la eternidad, y la conociera según su ser esencial y de existencia; entonces la conocería según su ser de esencia y de existencia, y ella no tendría sino un ser conocido - y esto como cuando se supone que no hubiera ninguna rosa, y que, no obstante, conozco a la rosa según su ser de esencia y de existencia aunque ella no tenga ninguno de éstos seres. De acuerdo con esto, el acto del entendimiento es el ser de la esencia o el ser de la existencia; sin embargo, aquello que se contrapone al intelecto como objeto de conocimiento, tiene solamente un ser disminuido en el intelecto. ${ }^{8}$

El objeto primario del conocimiento no se corresponde con el ser percibido, sino con el ser en cuanto es objeto del entendimiento, tal y como es aprehendido eternamente por el ser de Dios, como ser esencial y a la vez como ser existente. No obstante, para que haya verdad al esse inteligible requiere de cierta correspondencia o adecuación con la cosa real, existente, cuyo ser es autónomo, pre-existente al intelecto. De manera que la cosa es verdadera en cuanto realidad independiente, capaz de manifestarse y de ser asimilida por el intelecto:

$8 \quad$ "Ideo dico quod res ab aeterno non habuit esse verum essentiae vel exsistentiae, sed fundat relationem idealem secundum esse deminutum, quod habuit ab aeterno (quod est esse verum, distinctum contra esse essentiae et esse exsistentiae, sicut patet ex VI Metaphysicae): sicut si ponatur quod ego fuissem ab aeterno et quod ab aeterno intellexissem rosam, ab aeterno tunc intellexi rosam secundum esse suum essentiae et secundum esse existentiae; et tamen non habuit esse nisi cognitum, - sicut si modo rosa omnino nihil esset, intelligo rosam et secundum esse essentiae et exsistentiae, et tamen neutrum habet. Unde terminus intellectionis est esse essentiae vel esse exsistentiae, - et tamen illud quod obicitur intellectui, tantum habet esse deminutum in intellectu." (Scotus, 1966, p. 468-469). 
Si no hubiera ningún intelecto, aún así toda cosa estaría determinada por su naturaleza a manifestarse [esset nata se manifestare], según el grado de su entidad. Y este "darse a conocer" [notitia] es aquello por lo cual la cosa se dice que es conocida por naturaleza, no porque la naturaleza la conozca, sino porque, en virtud de su manifestación propia -mayor o menor- estaría determinada por su naturaleza, en lo que respecta a ella misma, a ser conocida de manera más o menos perfecta (Scotus, 1997, p. 66, trad. de Guerrero Troncoso).

El objeto primario del conocimiento es el ente, que cuenta con modos de ser propios, de manera que la verdad es resultado de la causalidad de las cosas extramentales y de su comprensión a partir del esse inteligible, del ser intencional. En relación con el objeto intencional, se puede decir que tiene un carácter atemporal, correlato del entendimiento. La rosa conocida, todos los objetos conocidos, existentes, son creatura intellecta (Cfr. Hoffmann 2002) que han advenido a la existencia gracias a la voluntad de Dios, quien los crea libremente, a partir de su representación proto-típica, ellos no se contraponen - no repugnan - la existencia. De este modo, el fundamento del conocimiento se remonta a Dios, quien piensa las creaturas, en su ser esencial e inteligible.

\section{Contraste con la fenomenología trascendental}

La diferencia con la fenomenología trascendental es palmaria, pues parten de dos concepciones distintas de las 'cosas' u objetos intencionales, del conocimiento y del mundo. Si bien el joven Heidegger encuentra motivos para plantear una lectura fenomenológica de la filosofía de Escoto, un examen detallado de su teoría de la intencionalidad indica que no hay muchos puntos de convergencia. En este sentido, tiene razón André de Muralt, quien ve en la teoría de la intencionalidad y en la ontología de Escoto, la raíz del objetivismo moderno. Paso a señalar las principales convergencias y divergencias entre estas dos versiones de la intencionalidad. En relación a la doctrina de la intencionalidad, existen tres puntos de convergencia entre Husserl y Escoto:

a) ambos pensadores liberan la lógica, cuyo carácter es conceptual, necesario, del campo de las ciencias empíricas como la psicología y las ciencias del lenguaje;

b) consideran la intencionalidad no cómo la recepción pasiva de formas del objeto, sino como una actividad consciente, en la que se dirige el rayo de la atención a un objeto intendido;

c) conciben la intencionalidad como una propiedad de la conciencia que no se limita a los actos teóricos, sino que se extienden a toda la actividad de la conciencia, a la sensibilidad y a la volición;

d) entienden el objeto intencional como el correlato objetivo de la tensión anímica, de la conciencia. De forma tal que basta con que la intención del acto se dirija a su respectivo objeto para que éste sea intencional (percibido, deseado, recordado, querido), de manera que para que algo pueda ser elevado al estatuto de 'intencional' no se requiere de que exista. El objeto intencional no está presente ni en el psiquismo, ni en la realidad extra mental, como cosa existente. En la V Investigación Lógica leemos: "Lo dado es para la conciencia exactamente igual, exista el objeto representado, o sea fingido e incluso un contrasentido. No nos representamos a Júpiter de otro modo que a Bismark, ni la torre de Babel de otro modo que la catedral de Colonia" (Husserl,1982, p. 495).

Las diferencias entre ellos, son en cambio, mayores:

a) La diferencia radical entre estas dos versiones de la intencionalidad reside en su interpretación del mundo, y con ello, de la trascendencia. Para Escoto existe una tercera región del conocimiento, adicional a la lógica y a la realidad natural, la objetividad inteligida. Dicha objetividad cuenta con diversos aspectos o sentidos, previos a la actividad del entendimiento, de manera que la realidad natural puede entenderse como una concreción a la que el entendimiento tan sólo accede indirectamente, a partir de los objetos inteligidos, intencionales. Considerar la objetividad como una dimensión neutra, allende la realidad física y lógica, que se corresponde con el campo metafísico de lo posible, le permite a Escoto desarrollar una teología que considera al entendimiento infinito, divino, como aquél que puede pensarlo todo, lo posible y lo imposible, de manera que todas las creaturas devienen existentes, gracias a que fueron previamente representadas en el entendimiento divino y a que él decidió crearlas. Para Escoto, el entendimiento puede acceder al patrón fundamental de la realidad, puede entender cualquier realidad, divina, terrenal o humana, real o posible, este parámetro es el 'ente'. Puesto que todo lo existente es inteligible, esto significa que todo lo que excede al pensamiento cae en el ámbito de la nada. Para Escoto, la ontología ofrece el instrumentario desde el cual se pueden pensar los atributos comunes a todos los seres, finitos e infinitos, los trascendentales. Si bien mediante la ontología no se alcanza a conocer a Dios en su ser personal, permite identificar su unidad, bondad, belleza. Aunque la metafísica no motiva la conversión religiosa, sirve de base para la comprensión del modo de ser de Dios y de la naturaleza.

La aproximación fenomenológica a la intencionalidad pasa por la criba de la reducción trascendental, de manera que el concepto de 'presencia' es diferente al de Escoto. Husserl entiende el objeto como un entramado entre ego-cogito-cogitatum, entre el yo, la manifestación sensible y la unidad objetiva, ideal. Cierto, la unidad constitutiva del objeto es resultado de la constitución subjetiva, mediante síntesis subjetivas, racionales, unidad ideal que sirve de polo regulador de las manifestaciones. De manera que la identidad del objeto permanece hasta nuevo aviso, en correlación con las múltiples manifestaciones. Es de recordar que para Husserl el aparecer es resultado de dos componentes correlativos: de una parte el "fenómeno" o manifestación, el componente vivencial y el 
componente objetivo. De manera que la cosa, aparece a través de los fenómenos, pero éstos no aparecen. $\mathrm{Al}$ respecto leemos en la V Investigación Lógica: "El fenómeno de la cosa (la vivencia) no es la cosa aparente, la cosa que "se halla frente" a nosotros supuestamente en su propio ser. Como pertenecientes a la conexión de la conciencia, vivimos los fenómenos; como pertenecientes al mundo fenoménico, se nos ofrecen aparentes las cosas. Los fenómenos mismos no aparecen; son vividos." (Husserl,1982, p. 478).

No obstante, Husserl no establece una dualidad entre la representación, intencional, y el objeto exterior, la considera como un contrasentido (Husserl, 1982, 530). De algún modo, Escoto comparte esta tesis en cuanto la relación entre la especie inteligible y el objeto, relación de semejanza, no está orientada hacia el objeto externo, existente, sino al esse inteligible, de carácter esencial. Para Escoto, el objeto singular es una haecceitas, inaccesible al entendimiento. No obstante, la fenomenología no asume la existencia de una realidad independiente e inaccesible a la experiencia vivida.

b) Dos concepciones distintas del "en tanto que" intencional. Para Husserl, el sentido del objeto surge del acto intencional, a tal punto que los contenidos de sensación sirven de base para la aprehensión intencional, de manera que, de una parte, el mismo objeto puede aparecer con diversos contenidos de sensación. Dos actos pueden aprehender un mismo objeto con base en distintos contenidos de sensación, así p. ej. un mismo tono lo podemos escuchar de cerca o de lejos. De otra parte, los mismos contenidos de sensación pueden aprehenderse de distintos modos. Los mismos datos sensibles pueden una vez ser interpretados de uno u otro modo, como sucede en las figuras propuestas por la Gestalt-Psychologie o con la equivocidad de las palabras (Husserl, 1982, 504). En este contexto, la aprehensión intencional se realiza teniendo presente los fenómenos y el horizonte en que aparece el objeto. En contraposición, el modelo escotista enfatiza la actividad del entendimiento que la de los actos de la conciencia, desconoce la distinción husserliana entre intenciones vacías y plenificación de sentido. Para la "distinción formal" escotista el sentido tiene un carácter eminentemente conceptual, de manera que cada atributo es una representación o forma mental que se asemeja - con una forma del objeto intencional.

Para Husserl, las vivencias, objetos inmanentes, no aparecen son vividas, permiten la remisión intencional; y los objetos trascendentes adquieren su sentido a partir de los actos intencionales, dóxicos, fundantes y fundados. El sentido objetivo proviene de horizontes subjetivos e intersubjetivos, históricos. Aun cuando el trasfondo inconsciente determine el aparecer, éste puede explicitarse mediante la reflexión racional. Husserl no concibe al objeto intencional como una realidad independiente de la conciencia, la intencionalidad no aprehende el objeto como algo exterior a ella, sino como su rendimiento, mediante la constitución intencional (Husserl, 1982, p. 537). Para los oídos de un fenomenólogo la afirmación escotista de que el objeto pre-existe a la concien- cia, en el entendimiento divino, es una pretensión de verdad metafísica, que no se puede someter a la evidencia de la donación, de la fenomenalización.

c) La fenomenología de Husserl se orienta por el programa de ser ciencia estricta, cierto, pero esto no significa que su eidética, su concepción de las esencias, se corresponda con una concepción del objeto como 'esse inteligible', dimensión inteligida, neutra, cuyo ser es independiente de la experiencia y de las operaciones de la razón. A primera vista, no habría gran diferencia entre la eidética fenomenológica y la doctrina medieval de las esencias. Sin embargo, Husserl no acepta que las esencias conformen una dimensión neutra, preexistente al acto intencional: para él, las esencias se constituyen en la conciencia como el proto-tipo universal que determina la comprensión de la fenomenalización. A diferencia de Escoto, y de las teorías de la intencionalidad como abstracción, Husserl afirma que la intencionalidad accede al objeto individual, singular, lo intuye. A las esencias no se accede mediante la abstracción intelectual, como objetos puros, ajenos al objeto singular, existente; Husserl rechaza la tesis medieval (Avicena, Enrique de Gante, Alberto Magno) según la cual las esencias conforman una dimensión neutra, ni singular ni universal. Por el contrario, para la fenomenología las esencias permiten, mediante la intuición, el acceso al objeto individual, el cual se concibe idealmente como unidad plena, en la cual no hay más aspectos por conocer. Al eidos se arriba mediante la ideación, en la cual participa la imaginación y no a través de la abstracción intelectual (De Muralt, 1985, p. 66-67). Sin embargo, en tanto idea, las esencias no fungen como mediación, no ejercen el papel de 'representación', sino que, en su proyección al infinito, desaparecen para darle paso a la intuición, el "principio de los principios".

Sobre esta base se desvanece la posibilidad de que la fenomenología acepte las 'intenciones segundas', tal y como lo proponen Tomás de Aquino y Duns Escoto. Para la escolástica, las intenciones segundas son resultado exclusivo de la actividad del intelecto, el cual mediante la reflexión accede a las formas intelectuales, mediante las cuales el pensamiento accede a la verdad. Los objetos de las intenciones segundas son entes de razón, los denominados universales, que aseguran el acceso a los individuos en dos pasos: en primer lugar, aseguran el acceso a la categoría primordial el sujeto (subiect) y a partir de él a las demás categorías. Sobre esta base se realiza toda la actividad judicativa. En el caso de Escoto, tales formas categoriales cuya base está en el concepto de 'ens' aseguran el acceso al objeto intencional. Univocidad del ente.

Para la fenomenología, el sujeto y el predicado, la sustancia y los accidentes, no son resultado de intenciones segundas, a ellos no se accede mediante un acto reflexivo, segundo, sino mediante la intención primera, de manera que la sustancia y sus atributos (accidentes) son correlato noemático de la experiencia, que se explicita luego mediante la actividad judicativa (De Muralt, 1985, 92-99). Husserl rechaza la tesis de la separación entre sensibilidad y entendimiento. Entre ellos, hay una gradualidad, de manera que las categorías no fungen exclusivamente 
como formas del pensamiento, sino que operan rudimentariamente en la experiencia pasiva, desde los niveles básicos de la temporalidad y de la sensibilidad, hasta las relaciones conscientes, efectuadas por el entendimiento. El objeto intencional, según la fenomenología, aparece sin la mediación de la especie inteligible, de la representación mental que permite la escenificación o manifestación del objeto, ofreciendo en cada casa un aspecto del objeto. En este sentido, Husserl estaría más cerca de Pedro de Olivi y de Guillermo de Ockham.

Esto lleva a dos concepciones de la 'presencia': de un lado, para la fenomenología, la presencia se realiza a través de la intuición de los diversos aspectos, vividos, mediante los cuales se manifiesta el objeto. Para Husserl, la fenomenalización resulta decisiva en la determinación de la unidad del objeto intencional. La intención funge al modo de una anticipación o mención vacía de los aspectos, los cuales pueden plenificar el sentido intendido. No obstante, la anticipación contiene siempre un plus irreductible, que no cubre plenamente la dación de la cosa. Esta es la base del zigzag, los aspectos objetivos están sometidos a un horizonte intencional, que se debe reexaminar con el fin de identificar capas de sentido ocultas, determinante de la comprensión del objeto. De otro lado, la filosofía escotista considera el 'en tanto que', determinante de la intencionalidad, como forma o acepción del concepto objetivo, que se adecúa a la cosa que se manifiesta o reluce en la especie inteligible. Si bien Escoto habla de una manifestación de la cosa, no da cuenta de cómo tiene lugar dicha manifestación, en la relación intencional, no se hace cargo de la fenomenalización, tal y como sí lo hace Husserl en la fenomenología. A Escoto le basta con afirmar que la manifestación de la cosa es posible simplemente porque le corresponde un modo de ser, el cual es asimilado por el entendimiento. De manera que la base del conocimiento, de la verdad, está en la capacidad del entendimiento de acceder al ser del ente, de permitir se que haga presente, en sus diversas modalidades, lo que lleva a Guerrero Troncoso a hablar de una "fenomenología de la verdad" (Guerrero, 2015, p. 89-90)

En contraposición, para Husserl la fenomenalización de la cosa no presupone el sentido de ser. El ser en cuanto ajeno a la conciencia es característico de la actitud natural, y por tanto, es puesto entre paréntesis. La reducción fenomenológica no parte del supuesto de que las cosas cuentan con un modo de ser ajeno a la conciencia, antes bien, la exterioridad del objeto, su trascendencia adquiere sentido a partir de la tomas de posición dóxica y de sus respectivos horizontes. La perspectividad de la intencionalidad está determinada por el observador, quien a partir de su facticidad, desde sus horizontes de mundo, temporales, corporales, intersubjetivos, otorga los diversos sentidos de ser. En cambio, la perspectividad escotista es resultado de la acción del entendimiento, que construye una representación mental, especie inteligible, en donde reluce el objeto intencional.

d) Es de recordar que Escoto entiende su filosofía como sciencia trascendens y Husserl como ciencia trascendental. Para el doctor sutil, los trascendentales son atributos divinos, por encima de los cuales no puede haber género alguno. Escoto examina a fondo el problema de la relación entre 'ser' y 'uno', y afirma, con Averroes, que esencialmente no son iguales, que no se pueden convertir libremente, aunque se refieran a un mismo sujeto, a un mismo sustrato. La scientia trascendens de Escoto permite acceder a las estructuras ontológicas de la realidad, a la unidad de todos los objetos, sin dificultad alguna. En cambio, para Husserl la unidad es un rendimiento de la actividad intencional, subjetiva, en distintos niveles, desde su origen en las síntesis pasivas de la temporalidad y de la sensibilidad, hasta los actos objetivantes o dóxicos, que determinan el ser del objeto intencional. Para la fenomenología la unidad no tiene un carácter lógico, formal, pre-existente a la conciencia, común a todos los entes, sino que es un rendimiento de la conciencia, en la constitución del sentido del ser. Esto permite afirmar que la unidad de la actividad intencional no funge al modo de una categoría, ni de un trascendental. No es una categoría porque ella no se aplica exclusivamente a un grupo de objetos, sino que está vinculada con la fenomenalización de todos los objetos; la unidad de la conciencia tampoco es un trascendental, porque - desde la perspectiva noética - es un rendimiento de los actos de conciencia y, desde el polo objetivo, noemático, la unidad pertenece a la idealidad del correlato, al objeto ideal pleno de sentido, al que apunta la serie de múltiples manifestaciones.

En suma, la filosofía escotista asume que a la realidad le corresponde el ser y que está divida, fácticamente, en diversos modos de ser (finito e infinito, esencial, real, representado). La verdad es correlativa al ser, que se manifiesta de diversos modos: de manera que lo falso correspondería al no ser. Ante esto surge la objeción de que la filosofía escotista presupone el ser de las cosas sin indagar por el origen de sentido del ser, por el fundamento de la verdad. En contraposición, en conexión con la tradición cartesiana, la reducción fenomenológica surge del intento de determinar la fuente del sentido del ser, es decir, por qué el ente que aparece como verdadero ante la conciencia lo es, independientemente de su aparecer fenoménico.

\section{Sobre el aporte de la scientia trascendens a la fenomenología}

Surge la pregunta de si a pesar de las diferencias, la scientia trascendens tiene algo que aportar a la investigación fenomenológica. Al respecto señalamos dos puntos:

En primer lugar, en relación con el concepto de 'intencionalidad', se puede afirmar que si bien la fenomenología no resuelve el problema de la intencionalidad, ofrece una concepción mucho más elaborada sobre los actos y objetos intencionales, en la medida en que indaga por el fundamento del sentido de ser, por los horizontes temporales y del mundo de la vida que determinando su génesis. La fenomenología ofrece mayores posibilidades para examinar el presupuesto de que hay una conexión entre ser y 
verdad. Si bien el idealismo trascendental husserliano tiene límites - es blanco de críticas con respecto a la tesis de que hay dimensión de la experiencia que no pueden ser determinadas plenamente por la actividad intencional - estas críticas permanecen en el marco de la fenomenología, de manera que no dan pie a posiciones realistas que liberan el sentido de ser a una dimensión totalmente independiente de los actos de la conciencia, ya sea en calidad de realidad natural, como dimensión inteligida, o como dimensión lógica, racional.

Para la fenomenología la relación con el ser se establece a partir de la constitución del sentido de ser. El ser no se revela con un sentido predeterminado, sino que cada cosa varía su sentido de ser en la experiencia, en la fenomenalización. Al ser no le corresponde una unidad pre-establecida, clausurada, sino que su sentido se constituye en la correlación intencional. Ninguna de las versiones del movimiento fenomenológico, aún las más críticas al idealismo trascendental, aceptaría la tesis de que la unidad y sentido del ser pertenecen a una dimensión previa a la correlación, a la manifestación. Desde esta perspectiva, el estudio de la scientia trascendens ofrece al fenomenólogo la posibilidad de aclarar la naturaleza y motivaciones de la constitución trascendental, tomar distancia de la lógica formal en favor de una lógica trascendental, explicación del origen del sentido de ser.

En segundo lugar, si bien no desarrollamos esta temática en este trabajo, cabe anotar que la metafísica escotista plantea preguntas vigentes sobre la relación entre lo sobrenatural y natural. La filosofía escotista aviva la pregunta de si los fines que rigen de la teleología son exclusivamente racionales y con ello se puede prescindir de Dios, o si por el contrario, Dios tiene el papel de dinamizar la teleología, desde su niveles primarios, pasando por los fines de la acción humana, ética, hasta los fines de la naturaleza. La pregunta de Escoto a los filósofos sigue vigente: ise pueden establecer, por medios exclusivamente racionales, las metas últimas de la existencia? A esta pregunta Husserl intenta dar respuesta exclusivamente a partir de la filosofía, sin embargo, no puede evitar fundamentar la ética en una visión personalista, del amor, con un trasfondo teológico, paulino, ineludible y tampoco puede evadir el misterio de la trascendencia divina, determinante del origen del mundo. Frente a las inevitables recaídas de la razón humana, la scientia trascendens escotista recuerda la importancia de repensar los vínculos entre metafísica y teología, motiva a retomar el sentido originario de la teología como ciencia práctica, orientadora de la acción humana, como ciencia que lleva a renovar el sentido misterioso y sagrado de la naturaleza, obra de Dios.

\section{Bibliografía}

Aristóteles (1994). Metafísica, Madrid: Gredos

Aristóteles (1999) Categorías. De interpretatione, Tecnos: Madrid, 1999
Avicena (1977) Philosophia prima sive scientia divina, i - iv », ed. S. Van Riet. Louvain-Leyde: Peeters-Brill

Brentanto, F. (1924) Psychologe vom empirischen Standpunkte, vol. I. Hamburgo: Meiner, 1924 (original: 1874)

Boulnois, O. (1999): Être et représentation. Une généalogie de la métaphysique moderne à l'époque de Duns Scot (XIIIeXIVe siècle), Paris: Presses Universitaires de France

De Muralt, A. (2008). La apuesta de la filosofía medieval. Estudios tomistas, escotistas, ockhamistas y gregorianos. Madrid: Marcial Pons

De Muralt, A. (1985). La métaphysique du phénomene. Les origines médiévales et l'élaboration de la pensée phénoménologique. Paris, Vrin

Guerrero Troncoso, H. (2015). “Duns Escoto y la noción de verdad concebida como fenómeno", en: Revista Philosophica, No. 47-48, pp. 59-89

Hoffmann, T. (2002). Creatura Intellecta: Die Ideen und Possibilien bei Duns Scotus mit Ausblick auf Franz von Mayronis, Poncius und Mastrius. Münster: Aschendorff

Honnefelder, L. (1990). Sciencia transcendens. Die formale Bestimmung von Seindheit und Relität in der Metaphysik des Mittelalters und der Neuzeit. Hamburf: FelixMeiner

Husserl, E. (1982). Investigaciones Lógicas, vol. 1 y 2, Madrid: Alianza

Husserl, E. (2013). Ideas relativas a una fenomenología pura y una filosofía fenomenológica. Libro primero. Introducción general a la fenomenología pura. México: F.C.E

Knudsen (1982). "Intentions and impositions", en Kretzmann et al. The Cambridge History of Later Medieval Philosophy, Cambridge University Press, Cambridge 1982, pp. 479-495.

Martínez Ruíz, C. (2014). “En los orígenes de la teoría escotista de la intencionalidad: Odón Rigaud y Pedro de Juan Olivi", en: Martínez R. (ed): Estudios sobre lenguaje y realidad en la baja edad media, Córdoba: Brujas, pp. $143-167$

Perler, D. (2001) “What are intentional objects? A controversy among early scotists", en: Ancient and Medieval Theories of Intentionality, Leiden: Brill, pp. 203-225

Pini, Giorgio (2002). Categories and logic in Duns Scotus. An Interpretation of Aristotle's Catego- ries in the Late Thirteenth Century. Leiden: Brill, 2002.

Pini, Giorgio (2008), Reading Aristotle's Categories as an Introduction of Logic: Later Medieval Discussions about Its Place in the Aristotelian Corpus", in: Lloyd A. Newton (ed.) - Medieval commentaries on Aristotle's Categories - Leiden, Brill

Salinas, H. (2016). La determinación de la lógica como ciencia común intencional en Duns Escoto, en: Franciscanum, No. 165 , p. $51-86$ 
Salinas, H. (2017). Naturaleza y unidad lógica de las categorías en Duns Escoto, en: Pensamiento, vol. 73, núm. 275, pp. $129-145$

Scotus, Duns (1997). Opera Philosophica III-IV., Quaestiones super libros metaphysicorum Aristotelis, New York: The Franciscan Institute

Scotus, Duns (1954). Opera Omnia, Ordinatio I, dist. 3, Vol. III, Ciudad Vaticana: Typis Polyglottis Vaticanis

Scotus, Duns (1960). Opera Omnia, Lectura, dist. 1-7, Vol. XVI, Ciudad Vaticana: Typis Polyglottis Vaticanis

Scotus, Duns (1966). Opera Omnia, Lectura, dist. 8-45, Vol. XVII, Ciudad Vaticana: Typis Polyglottis Vaticanis.

Julio César Vargas Bejarano es Profesor Titular del Departamento de Filosofía de la Universidad del Valle, Cali (Colombia). Email: julio.vargas@correounivalle. $\underline{\text { edu.co }}$

Recebido em 23.01.2018

Aceito em 12.06.2018 